\title{
Psychological Impact of Isolation from Leader in Virtual Teams on Team Interaction
}

\author{
Shivam H. Shah \\ Allen D Nease High School, 10550 Ray Road, Ponte Vedra, Florida, 32081,USA ; Shivamshahfl@gmail.com
}

\begin{abstract}
The purpose of this study was to develop a model to show how isolation from a leader can affect employee relationships and the perception of social loafing in virtual teams. This model, assessed through qualitative interviews with virtual team employees, suggests that the effects of isolation vary from employee to employee based on the title, internal motivation, and surrounding environment. However, this model predicts that these employees will not only worsen their relationships with their teammates in the transition to a virtual setting, but they will also experience reduced relational closeness and increased uncertainty. These effects will cause employees to experience more work fatigue, put in less effort, and contribute fewer ideas (an increase in corresponding social loafing). Conversely, this research also proposes that companies are taking an increasingly active role in preventing and solving these issues to hinder the spread of these effects. The implications of these phenomena are discussed in this study..

KEYWORDS: Behavioral and Social Sciences; Sociology and Social Psychology; Employee Relationships; Virtual Teams; Social Loafing.
\end{abstract}

\section{- Introduction}

Before the effects in the workplace are explored, it is important to define the relevant terms. The employee relationship is defined as the relationship between two or more employees, measured by collaboration and communication. Similarly, virtual teams are teams that work towards a common organizational goal primarily by utilizing technology in a virtual setting - such as Zoom, Skype, or Microsoft teams. Social loafing is often defined as the "the tendency of individuals to contribute less in a team setting" or in other words, the tendency to put in less individual efforts in a group setting due to the collective measurement of performance. ${ }^{1}$ This phenomenon causes decreased productivity and demoralization of the hard working members that witness these members starting to complete less work. If employees begin to complete less work, organizations have less information from which to make decisions, compromising the accuracy of their decisions while hindering the advancement of the team as a whole. These varying definitions and effects will help to answer the question and narrow the scope for the interviews to ensure that the sample size is representative of the population.

The implications are numerous. If virtual teams increase team interaction and decrease social loafing, teams that have the option to work physically may switch to the virtual format, saving the company thousands of dollars in operating expenses. Moreover, researchers have previously studied the overarching topic of virtual teams, but the connection to the leader is rarely researched; further research on this connection will allow us to understand the leader's role in team interaction and social loafing. ${ }^{1}$ Social loafing is prevalent in virtual technology because it allows employees to hide behind the screen and avoid connecting with their teammates - even if they have no prior teamwork experience - or will not work with this virtual team after the pandemic. If employees put in less effort, team spirit and the overall productivity of the company is affected. However, results reflecting team interactions have been inconclusive as studies have stated that teams can bond more successfully depending on the tool used and the involvement of the leader. The degree of virtuality, and other team variables such as current stage, experience, and past involvement have also been discovered to play a role. However, researchers still fail to understand how distance from the leader specifically affects social loafing and team interactions.

To answer this question, we conducted a qualitative, interview-based study entailing five employees across the United States that work in virtual teams at different companies. After recording and analyzing their responses on how the isolation from the leader has affected their interactions, relationships, communication, collaboration, and contributions, we deduced the emergent patterns that materialized. Responses were expected to indicate whether the team factors increased or decreased with the team's shift to a virtual setting.

\section{Conceptual Framerworks:}

Social loafing is present in the online setting, not just in knowledge teams or technology-supported teams. Past research attributes the phenomenon to the increase in cognitive disengagement mechanisms in virtual teams: diffusion of responsibility, attribution of blame, and dehumanization. ${ }^{1}$ Additionally, the overall structure and work environment in tech-supported industries facilitate social loafing. Employees frequently need to mentally justify the feasibility of their ideas before stating the ideas in the brainstorming phase. This creates a deficit of solid, potential ideas. In the end, this study found that dehumanization is a considerable mediator in social 
loafing in virtual settings while the other two variables are mediators to a much smaller extent. ${ }^{1}$

A major step into the research of physical isolation from the leader was a preliminary study conducted on the leader-distance theory. ${ }^{2}$ The researchers found that this distance is categorized as "leader-follower physical distance, perceived social distance, and perceived task interaction frequency". This study will explore the physical and social isolation from leaders. In technology-mediated environments virtual communication is often an asset to an organization's leader. Other times, it is "noise" to the leader's speech and modeling behaviors, which can decrease the effect of a leader's inspiration on his or her employees. If a leader fails to motivate and inspire their employees, and the team refrains from partaking in "identifying responsibilities of team members, maintaining frequent contact, and promoting team-related aims," a permanent erosion in trust and relational closeness could appear, leading to the negative exacerbation of team relationships, collaboration, and communication. $^{2}$

Furthermore, researchers found that when task visibility was low, social loafing was more likely to occur due to the level of intrinsic motivation serving as the moderator. ${ }^{3}$ This implies that in organizations and virtual teams that have low task visibility, there needs to be higher levels of intrinsic motivation, like "task significance, task meaningfulness, [and] task contribution" to compensate and boost motivation. ${ }^{2}$

To elaborate, communication can be affected depending on its medium and type. ${ }^{3}$ For example, new types of technology should aid employees in fostering relationships and creating a sense of cohesion and collaboration. However, the "lean, asynchronous, communication environments" that many companies are implementing coupled with the "temporal coordination mechanism" seem to not only positively impact team relationships but also seem to prevent animosity through virtual interactions and aid conflict management.

Past research on social loafing demonstrates that virtual teams have functioned in a linear structure, suggesting that the factors are not interdependent. However, current research has proven a configurational perspective in which one effect may magnify the effect caused by another variable. For example, if team identity was present, it would magnify the level of effective communication and vice versa. If certain constructs such as team identity, collaboration, and communication are investigated, teams can transition into high-performing teams and increase relational closeness while decreasing the presence of social loafing. ${ }^{4}$

Similarly, social loafing can only be measured through self-feedback, but participants are not very aware of its presence. Past studies furthered research on the factors that contributed to the high level of social loafing. ${ }^{5}$ If the virtual team has not worked together in the past or will likely dissolve after the pandemic, employees will believe that the arrangement is temporary. This belief causes lower levels of motivation and corresponding increases in social loafing. However, researchers believed that this decrease could be mitigated with feedback to increase productivity. While productivity does increase with feedback, the presence of social loafing still permeates virtual teams. ${ }^{5}$

A study explains social loafing from the perspective of mediators. Past research suggests that social loafing is affected by the familial responsibilities (the amount and type of duties that employees have in their families) and the team composition (the relative familiarity and likeliness of members in a team). The trend suggests that the higher the number of family responsibilities, the higher the degree of social loafing- although the cohesion and psychological obligation may regulate this higher level. Nonetheless, teams can still bond and familiarize themselves with each other virtually, but family activities may take precedent. In this case, due to the seniority and of the interviewees, corresponding with higher familial responsibilities, the results may be slightly skewed towards the presence of social loafing. ${ }^{6}$

Furthermore, the ability of employees to work with socially similar - common levels of familial responsibilities and approach to work - workers has a profound influence on their cohesion and connection to the rest of the team. "Carefree individuals who worked with mostly busy teammates perceived low levels of cohesion and obligation to their teammates and reported increased levels of social loafing in the team as virtuality increased," but this trend is reversed when a busy teammate was to work with primarily "carefree" teammates. The extent of social loafing could vary based on the workers and their environment. ${ }^{6}$

Amidst the current pandemic, it will be interesting to see how the emergent of new conferencing and communicating technology such as Zoom, Skype, and Microsoft Teams will affect communication in collaboration. ${ }^{7}$

\section{Methods}

The research design is an interview-based study within which I interviewed virtual team employees to create a conceptual study on the topic. First, I reached out to contacts in varying sectors - banking, technology medicine to request an interview in July 2020.

The interview questions, presented in Table 1, were purposely open-ended to allow for more qualitative and open responses, and to capture other unnoticed trends (would not be possible with a quantifiable evaluation). Questions 3, 7, and 8 were intended to reveal the perception of social loafing from different angles and questions 2, 3, and 5 were intended to reveal the relationship between different employees (Table 1). Furthermore, questions 1,4 , and 6 were intended to capture other trends and effects of the transition that are connected to the isolation and disconnect in a virtual work environment.

Afterward, I called the various employees and conducted interviews while recording their responses through the voice memos feature to ensure that no information was unaccounted for. Then I transcribed the audio recording and analyzed the data to find potential patterns. The recording was necessary to avoid psychological biases and avoid any biases or misunderstandings in the study based on my hearing capabilities and listening comprehension. However, some questions had to be quantified to gauge the overall change between the 
pre-COVID physical setting and the virtual setting, in which employees were isolated from their leader.

Table 1: Interview questions that were asked to the interviewees.

\begin{tabular}{|ll|}
\hline 1. & How do you think isolation from the leader impacts your interaction and relationships with your \\
teammates?
\end{tabular}

For the experiment, I was able to interview 5 employees at varying companies. Although this number is on the low end for the sample sizes, I used a random number generator to select these 5 employees off of a list of 50 employees. The original purpose was to establish the presence of this common phenomenon so, with 5 employees, this study will be able to pass the population proportion and the results can be considered statistically significant. The first employee was a software engineer at the highly coveted Microsoft. Due to the unique and flexible team structure at Microsoft, his perspective on the issue may be limited. Next, I interviewed a software engineer at Google. However, Google's organizational structure could impact team dynamics differently as there are multiple supervisors for one virtual team. Teams are created with some managers being leaders in some teams and just employees in others. Last but not least, I interviewed an executive at Mckinnison. His perspective may also be limited due to past involvement with his virtual team and the inherent global dispersion of team members before the pandemic - team members were already scattered all across the globe. The next member was an executive member at Pinnacle Capital Management. His perspective may be limited by the seniority of his position as president/CEO. The last was a cancer researcher for Baptist Hospital.

\section{- Results and Discussion}

Through these interviews, several emerging factors and patterns revealed the true impact of the isolation on employees. First, based on the isolation from the leader, employees seemed to endure a lack of morale and a sense of accountability. The leaders seemed to play an instrumental role in connecting team members and cultivating morale and accountability. When workers converse about new ideas and their implementation, this culture of innovation and critical thinking holds them accountable to innovate and brainstorm ideas of their own. For example, one employee stated that he misses the "ad hoc conversations or the water cooler conversations" ${ }^{\prime}$ that allowed him to connect with the group and thus, boost his effort. Due to the isolation from the team leader, the soul of the team, this sense of accountability seems to play a mediating role to weaken efforts.

The first trend is that the research exposed was how isolation magnifies the disconnect between employees. Employees at different companies state that they are transitioning from "work buddies to more of just colleagues or coworkers". ${ }^{9}$ Thus, team interactions and relationships continuously diminish in this isolated environment. Previously, the leader had played a connecting role by hosting team meetings that fostered employee-employee relationships, collaboration, and communication among team members. In the virtual format, companies are hosting fewer meetings and the leader plays less of this connecting role that is so vital to improving employee relationships and decreasing social loafing.

The second trend is that communication and collaboration have decreased but asynchronous communication has increased. Due to the nature of virtual teams, isolation from the leader has disconnected employees and caused resulting decreases in communication and collaboration; however, communication through asynchronous forms such as email and chats have increased, even pervading into after-hour periods: "beyond the regular hours from 8-5." 9 Maybe because of the increased uncertainty about tasks and less interconnectedness, employees are taking longer to discuss issues and find solutions. Virtuality has slowed down processes and has negatively impacted team interactions, costing employees and companies higher overhead in the process.

Furthermore, there was an increase in work fatigue when working from home. Due to the fact that communication is increasingly difficult and occurred at odd times, workers are exposed to their work more than they would be regularly. One employee even stated that employees have to work more to achieve the same amount of work rather than if they were in person and sitting in a quiet environment as the "overhead is a lot to achieve the same amount of work". ${ }^{9}$ In other words, this extended isolation has increased work fatigue - another moderator in the study - and taken a toll on them both physically and mentally, indirectly negatively affecting their relationships with other teammates and motivation. Thus, this effect causes higher levels of effects of isolation and social loafing.

The fourth trend was that in the virtual format, team members were less motivated and were only motivated when held accountable by performance evaluation standards of their jobs. This trend could be explained by the external environment and distractions, lack of accountability and morale, or even the lack of collaboration and communication in the virtual setting. However, interestingly the dip in motivation was very minor, only about "20-25\%" according to past employees. ${ }^{9}$ This may be due to their jobs and teams' prior relationships, but this facet needs to be explored more in-depth as both teammates showed slight decreases in their motivation: one from a "9 or 10 " to an " 8 or 7" 9 and from the " $8-10$ " range to the " $7-9$ " 10 range. Performance evaluation standards were also moderating factors as they ensured that teammates had to collaborate and communicate, increasing team relationships, and decreasing the likeliness of social loafing.

Similarly, the time after the transition to a virtual setting when the impact is measured also seems to have a distinct effect on the impact. Employees have stated that it depends on the team: some have said that "longer this goes on the more people are shying away from innovation or ideating or executing on new idea" 9 while some have found it "quite destructive in the beginning of the whole process". ${ }^{10}$ Depending on the team stage and time measured, the results of the isolation and 
the role that the leader plays can have a varying effect on the group. Thus, some teams in the performing stage have adapted and maintained levels of collaboration and communication while others in the norming stage have seen the effects of isolation magnified in their teams with worsened collaboration, communication, and relationships.

The sixth trend was that past involvement in the virtual team can affect the impact of isolation as well. This moderating variable can impact the motivation and contribution of ideas but not directly impact the relationship between teammates or the presence of social loafing. For example, teammates that had been geographically scattered before the COVID-19 pandemic experienced less of an impact from the isolation; one teammate stated that "normally the users are scattered for me and so for me, my team is scattered all over the place". ${ }^{10}$ Furthermore, the interactions in a team can vary based on the ability of the team to work together and motivate each other: "workers need a good team to motivate each other and clearly know what the objective is". ${ }^{11}$ Without a supportive team, team members may not be held accountable or included, diminishing their motivation, and thus relationships while increasing social loafing in virtual teams. This effect could also be magnified or diminished based on the personality and individual motivation of each person on the team. Organizational structure impacts isolation as well. The flat organizational structures in Google and Microsoft compared to tall organizations in Boeing or Toyota are noteworthy because flat organizations are less likely to experience this effect of the isolation from the leader, demonstrated in the slightly more connected reviews of the employees that were interviewed. As one employee stated, "depending upon different personalities, some people really find it hard to communicate our concentrate in our conversations" ${ }^{9}$ which shows that extroverts or not our may be compatible and be less motivated with the online format. Others that are not technologically savvy may shy away from the use of technology to collaborate or communicate, diminishing these inter-team employee relationships and increasing social loafing. Employees that are internally driven, "whether they work at home or office they will do what it takes to get what needs to be done." ${ }^{11}$ For these employees, the isolation's impact might not affect them to the extent that it affects other employees.

The seventh trend was that the environment seemed to have mixed consequences on team interactions and social loafing. Because many employees had high levels of household responsibilities, they spent less time on their work. Coupled with the lack of direct oversight from the leader, the employees were found to be more distracted in their work. However, some employees found this time at home to be an opportunity to work "with less distractions" ${ }^{2}$ and focus more on work and put in longer hours. The employees that were internally driven seemed to put aside time and a quiet space for work to surpass their performance goals. Thus, the moderator of the external environment and distractions seem to result in inconclusive evidence on team interactions and social loafing due to the differing motivations and responsibilities of employees at each company. The moderating variable of hours worked also has mixed effects- bridging employees, and closing gaps in collaboration and communication, or contrarily causing work fatigue.

However, to minimize the damage done through isolation, I have found that many companies use technology to foster communication and collaboration in virtual settings. In the $21^{\text {st }}$ century, with the advent of new group communication and collaboration technologies such as Zoom, Skype, and Microsoft Teams, these technologies' implementation would be critical more than ever. When implemented, "due to the ability of the video calls and other technological innovations, me and my teammates try to stay in touch." ${ }^{12}$ It seems as if the collaboration and communication have been bolstered and have not taken major hits just because of the extended virtuality. Thus, across the board technology — as a moderator- has helped teammates to interact and decreased the chances of social loafing- social loafing has been found to been present in virtual settings regardless of team, jobs, or employees.

Not only has communication and collaboration decreased in the workplace, but there have been paralleled negative effects in "sharing of the ideas". ${ }^{12}$ In the virtual format, employers can witness "more struggle[s] or roadblocks" in the innovation process, decreasing the mediating mechanism of the individual contribution of ideas. ${ }^{12}$ If individuals do not contribute ideas readily, the presence of social loafing increases as employees do not want to put in more effort in the face of these roadblocks of the virtual environment. The individual contribution of ideas serves as a mediating mechanism on employee relationships and social loafing. If ideas are more willingly contributed to the virtual environment, employees are more open to contribute their own ideas, increasing connectedness with the team and decreasing social loafing. They also believe that ideas are more permanent online because they become inter-departmental in conferences, so they believe that they are obliged to ensure that their ideas are feasible and reasonable before they contribute. Finally, the brainstorming environment online is not comparable to that of the physical setting "ideas triggering off one discussion and something else is much more effective in the workplace versus in a virtual environment." 11 If workers feel that their opinions will be less valued in the virtual environment and cannot even be implemented, due to the companies shutting down many projects, they will see it as futile and put in less effort in the other sectors which is indicative of social loafing.

The last trend was that employees during COVID-19 have felt more uncertain in their relationships with each other and their managers due to a loss of physical connection that was present in the workplace. This has increased the presence of social loafing. There is also uncertainty about work status, incoming tasks, and their manager's perception of their performance. In the virtual workplace, "People are also more fearful, and they do express uncertainties," reinforcing the divide between employees. ${ }^{12}$ This divide causes employees to put in less work if they are not sure how their manager will perceive it, or they may have to redo or modify the project. Thus, there are increases in social loafing and decreases in the collaboration and communication between employees because of the uncertainty of the work aspects of the transition into a virtual environment. 
However, many companies have acknowledged this mediating variable of individual motivation and uncertainty and have taken a proactive response: they implemented controls to ensure that workers still complete their tasks while motivating them to work towards their goals. Some companies have opted to put major projects on hold so that they do not lose money from the inactivity and increased virtual overheard while others have tried to maintain the previous levels of collaboration and communication through "regular weekly and daily meetings...if people have any issues or concerns, they can raise them, and we can solve them". ${ }^{13}$ This development at least shows that even though evidence points to an increase in social loafing in the virtual format and a decrease in collaboration and communication, with the exception of asynchronous communication, companies are taking steps to increase employee motivation, happiness, and commitment throughout this challenging, virtual work environment.

\section{Limitations:}

Despite its interesting results, this study has several limitations worth mentioning. In other industries, especially blue-collar jobs, one may observe higher effects of isolation in communication and collaboration due to the more involved and authoritarian role of the leaders in those jobs. On the same note, cultural influences could influence personal motivation levels that could play a role in explaining the high levels of internal motivation, prompting lower levels of leader-follower isolation than others, and causing workers to remain motivated and connected with the rest of the team. Thus, in consideration of all of these factors, my study may slightly underestimate the true impact of isolation on collaboration and communication. First, the inclusion of younger workers and workers from other cultures may increase the effect of isolation on collaboration and communication and magnify the growing distance between employees through technology. Companies that did not heavily utilize technology before COVID-19 may lose out on the collaboration and communication that is needed for daily tasks. Lastly, since more experienced workers could have experienced a prior sense of connection and possess prior team experience, younger workers could experience the effects of diminishing collaboration and communication much quicker and stronger than the experienced workers in this study. Further research could build off of the research of the representative sample conducted in this paper and seek to corroborate these results.

\section{- Conclusion and Implications}

Future research on the topic is suggested to follow a certain pattern to garner more generalizable results. First off, in the future, it would be useful if a follow-up quantitative study was performed using randomization and a large population.

In light of the research, if companies do not act on the growing divide between employees, technology, and organizations, then social loafing will continue to increase, and employee relationships will continue to worsen. The first conclusion I deduced was that collaboration and communication have decreased with a parallel divide between employees in virtual teams and organizations. Because employees may not be familiar with virtual tools or are less motivated, communication decreases as it requires more effort to communicate the same message than in a virtual format. However, there are varying effects of this process on employees. As each employee has a different level of motivation, experience, and title, the effects of isolation such as the lack of collaboration, communication and the increase in social loafing still occur, but on different levels. Differing virtual work environments may pose more or fewer distractions and different learning styles may affect the work experience as well. However, in all scenarios, employees reduced their efforts in virtual settings. The lack of morale and motivation, coupled with the lack of a sense of accountability, contributes to the magnification of these effects. As employees possibly are less motivated and encouraged by teammates compared to the physical format through ad-hoc conversations or meetings, they are less likely to experience a sense of connection with the team and put in the necessary effort to productively function as a team.

Furthermore, employees are experiencing reduced relational and interpersonal closeness. With increased virtuality comes fewer opportunities to interact, enlarging the divide between employees. Moreover, because asynchronous communication requires both employees to talk at the same time and employees usually do not reply immediately, employees converse less often than in the physical format and have limited the conversation to work-related matters. Thus, team-wide outings and events have decreased, reducing the closeness between employees as they transition from friends into colleagues. Due to the reduced closeness and other effects of virtuality described in the paragraph above, employees are less likely to contribute ideas. This research demonstrates that employees believe that their ideas are not feasible in this time and since a declaration or introduction of ideas means that ideas are distributed officially to the entire team instead of a brainstorm, the details may have to be fully planned before. Employees may not be willing to go this extra step and think of the details, contributing to the lack of new ideas during this COVID-19 period. All of the conclusions above and in the first paragraph have contributed to the increased work fatigue in the virtual format. Employees are working more but achieving the same amount of work, increasing their work fatigue. This lack of motivation and relational closeness as well as the also increasing social loafing and reluctance to put in the extra effort has also caused them to encounter higher levels of work fatigue.

Armed with these facts, businesses have been found to take an increasingly involved role in mitigating these effects. From implementing productivity controls and performance standards to creating opportunities and resources for employees to interact, businesses are ensuring that teams can still function productively while developing relationships and contributing ideas - hence, reducing social loafing in virtual teams. Consequently, another conclusion that was found is that companies cannot fully and effectively manage their employees virtually, so they are relying on technology to do so for them - thus, automating the process and providing employees with the tools they need to succeed. Tools such as Zoom, and Slack are at use at an all-time high to preserve the level of communication, 
collaboration, and introduction of ideas that occurred in the physical format.

In light of these conclusions, this research has implications for many aspects of management. First, companies are and need to continue taking advantage of the $21^{\text {st }}$-century collaboration and communication tools to ensure that collaboration and communication does not diminish in the transition to virtuality. These technological tools - Microsoft Teams, Skype, and Zoom - need to be extended to all employees so that employees communicate and collaborate. In this transition, virtual team leaders may need to play a more involved role by involving themselves in the matters of the team so that they can reduce relational distance. Companies could even host virtual events or meetings to make up for this phenomenon. Furthermore, employees do not perceive unrefined ideas as implementable and thus refrain from introducing these ideas. In the virtual format, managers may need to encourage idea-sharing and even create certain times and places to share these ideas, such as brainstorming sessions.

Furthermore, because of the increased after-hour collaboration and communication in a virtual setting, managers may need to implement procedures to restrict work after hours to decrease work fatigue from the increased asynchronous collaboration. If employee efforts can be harnessed during the work period, not only will productivity increase, but work fatigue in the virtual format will decrease. However, because research has shown that the effects of the virtual environment can vary from employee to employee and organization to organization (highly motivated individuals may persevere regardless of the environment), leaders may need to take more of an individualized approach within the team to ensure that all employees-even the ones that are not that familiar with the virtual environment - can keep up and succeed. This study adds to the growing knowledge about management as it investigates and confirms the presence of these isolationary effects in organizations amid this switch to a virtual format, prompting organizations to take mitigative actions.

However, as there was an employee that seemed to adapt well to the online environment, it can be said that the effects of virtuality may vary by person. Employees that enjoy working at home, such as introverts and employees that are more familiar with the use of technology, may harness online tools to reduce its effects and stay on pace. Thus, even amidst this shift, some employees have independently taken efforts to stay on track.

\section{Acknowledgements}

I cannot express enough thanks to my mentor, Professor Nataly Lorinkova of the Mcdonough School for Business. I offer sincere appreciation for the learning opportunities provided by her and her continuous guidance throughout this writing process and as well as her continuous instruction on virtual teams in organizations.

\section{References}

1. Perry, S. J., Lorinkova, N. M., Hunter, E. M., Hubbard, A., \& Mcmahon, J. T. (2016). When Does Virtuality Really "Work"? Examining the Role of Work-Family and Virtuality in Social Loafing. Journal of Management, 42(2), 449-479. https://doi.org/10.1177/0149206313475814

2. George, J. M. (1992). Extrinsic And Intrinsic Origins Of Perceived Social Loafing In Organizations. Academy of Management Journal, 35(1), 191-202. https://doi.org/10.2307/256478

3. Montoya-Weiss, M. M., Massey, A. P., \& Song, M. (2001). Getting It Together: Temporal Coordination And Conflict Management In Global Virtual Teams. Academy of Management Journal, 44(6), 1251-1262. https://doi.org/10.2307/3069399.

4. Zimmermann, A. (2011). Interpersonal relationships in transnational, virtual teams: Towards a configurational perspective. International Journal of Management Reviews, 13(1), 59-78. https://doi.org/10.1111/j.1468-2370.2010.00284.x

5. Suleiman, J., \& Watson, R. T. (2008). Social Loafing in Technology-Supported Teams. Computer Supported Cooperative Work (CSCW), 17(4), 291-309. https://doi.org/10.1007/s10606-008-9075-6.

6. Alnuaimi, O. A., Robert, L. P., \& Maruping, L. M. (2010). Team Size, Dispersion, and Social Loafing in Technology-Supported Teams: A Perspective on the Theory of Moral Disengagement. Journal of Management Information Systems, 27(1), 203-230. https://doi.org/10.2753/mis0742-1222270109.

7. Marks, Michelle A., Mathieu J.E.,Zaccaro S.J. "A Temporally Based Framework and Taxonomy of Team Processes."The Academy of Management Review, vol. 26, no. 3, 2001, p. 356., doi:10.2307/259182.

8. Antonakis, J., \& Atwater, L. (2002). Leader distance: a review and a proposed theory. Leadership Perspectives, 129-160. https://doi.org/10.4324/9781315250601-11.

9. Shah, S. Google. Personal communication, July 2020.

10. Bhansali, P. Pinnacle Capital Management.

Personal communication, August 2020.

11. Shah, M. McKinnison. Personal communication, July 2020.

12. Shah, J. Microsoft. Personal communication, August 2020.

13. Gajarawala, N. Mayo Clinic. Personal communication, August 2020.

\section{Author}

Shivam Shah, the author of this research, is in his senior year of high school senior in Jacksonville, Florida. After taking several business classes at a local community college and taking accredited courses at other university, he decided to further his passion through research and application. 